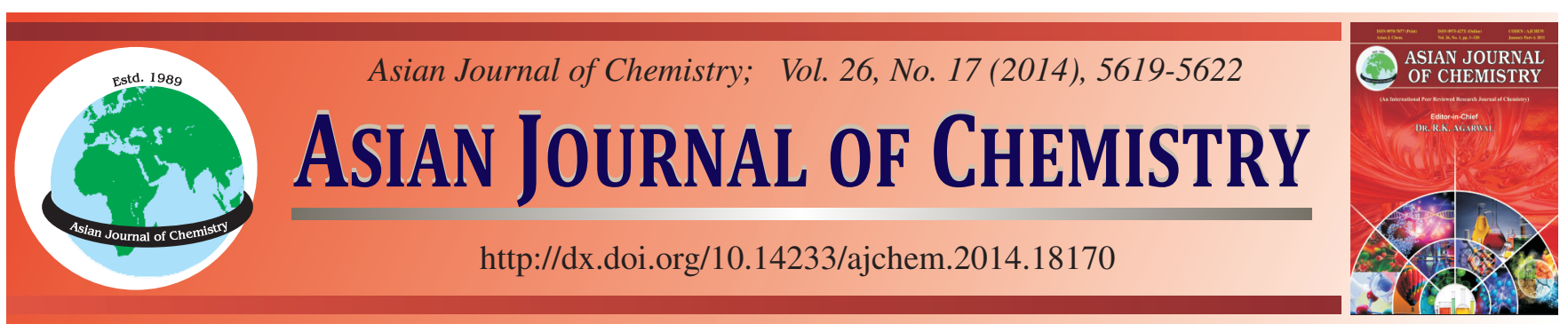

\title{
Pseudomonas cepacia Lipase Immobilized onto Chitosan-Coated Activated Carbon: An Efficient Catalyst for Transesterification Enantiomer Resolution of (R,S)-1-Phenyl-3- buten-1-ol $\dagger$
}

\author{
Ping Xue, Weiguang Su*, Weijing Ye, Tiansheng Zhao and Xiaoyong Lai
}

State Key Laboratory Cultivation Base of Natural Gas Conversion, Ningxia University, Yinchuan, P.R. China

*Corresponding author: Fax: +86 951 2062323; Tel: +86 951 2062835; E-mail: weiguangsu@nxu.edu.cn

Pseudomonas cepacia lipase (PSL) immobilized onto chitosan-coated activated carbon (CS-AC) with specific surface area of 798 ( $\left.\mathrm{m}^{2} / \mathrm{g}\right)$, average pore diameter of $2.13 \mathrm{~nm}$ and pore volume of $0.43 \mathrm{~cm}^{3} / \mathrm{g}$ was synthesized by a simple adsorption method. The immobilized lipase PSL/CS-AC catalyst exhibited enhanced catalytic activity for resolving racemic 1-phenyl-3-buten-1-ol compared to free PSL and PSL immobilized on sole activated carbon or chitosan. The enantiomeric excess value (ee $)$ of (S)-1-phenyl-3-buten-1-ol and the enantiomeric excess value (ee $\mathrm{p}_{\mathrm{p}}$ ) of (R)-1-phenyl-3-buten-1-ol acetate reached 99 and $94 \%$, respectively with the reaction substrate conversion of $51 \%$. PSL/CS-AC immobilized lipase exposed the more surface active sites resulting in the higher catalytic performances. The preparation of the support for immobilizing lipase is low cost and the reaction conditions are moderate and environmentally friendly. The immobilized lipase PSL/CS-AC catalyst possessed of high thermal stability, reusability and storage stability, it could be a potential chiral catalyst for enantiomer resolution reactions.

Keywords: Pseudomonas cepacia lipase, (R,S)-1-Phenyl-3-buten-1-ol, Immobilized lipase, Chitosan-coated activated carbon.

ᄂ - - - - - - - - - - - - - - - - - - - - - - - - - - - -

\section{INTRODUCTION}

Enantiomerically pure alcohols and esters are important intermediates for synthesizing fine organic chemicals and widely used in the pharmaceuticals, agrochemicals and chemical engineering ${ }^{1,2}$. Optically active 1-phenyl-3-buten-1-ol and its substituted derivatives are important precursors for synthesis of enantiomerically pure natural compounds and biologically active molecules, such as tetrahydrofuran, $\alpha, \beta$-unsaturated lactones, antitumour macrolides, alkaloids, chiral epoxy alcohols, $\beta$-hydroxy carbonyl compounds and optically pure 1,3-diols, etc. ${ }^{3-5}$. Efficient transesterification resolution of racemic alcohols is one of the most important reactions to produce optically pure alcohols and esters. In comparison with classical chemical methods such as preferential crystallization, chromatographic separation, biocatalytic processes are broadly accepted as good options to prepare optically pure alcohols ${ }^{6}$. Lipase is one of the important biocatalysts and widely used in the synthesis and resolution of high optically pure chiral compounds $^{7,8}$, which is mainly based on its high efficiency, stereoselectivity, mild reaction condition and environment friendly, etc. However, free lipase is difficult to be widely used in industry because of its insufficient stability, easy deactivation as well as hard recovery and re-use. In this case, immobilization of lipase can availably overcome these applied limits ${ }^{9,10}$ and it has been a hotspot in lipase catalysis.

Usually, the properties of immobilized enzyme depend on the nature of the support ${ }^{11}$. Hence, it is important to develop an efficient support to improve enzymatic properties of immobilized enzyme. Activated carbon (AC) is one of the most promising supports for enzyme immobilization because of its large surface area, high adsorption efficiency ${ }^{12-14}$. However, the enzyme with a benign surrounding microenvironment cannot be provided by simple physical adsorption between enzyme and activated carbon due to low surface $\mathrm{OH}$ groups density on activated carbon. Therefore, many investigations have focused on the surface modification of activated carbon with organic functionalities to improve enzymatic properties ${ }^{15,16}$. Chitosan (CS), the amino polysaccharide has received much attention because of its low toxicity, good biocompatibility, physiological inertness, remarkable affinity to protein and easy availability of its reactive amino and hydroxyl groups ${ }^{17,18}$. Chitosan was used as a modified material to improve the surface biocompatibility and increase the surface functional groups density of the activated carbon.

In the present work, the surface of activated carbon was modified by the natural polymer chitosan with a physical 
coating method, which was denoted as CS-AC. The lipase (Pseudomonas cepacia lipase, PSL) was successfully immobilized on the CS-AC and used for transesterification resolution of (R,S)-1-phenyl-3-buten-1-ol to obtain high-purity chiral single enantiomers. The immobilized lipase PSL/CS-AC showed the highest catalytic activity and enantioselectivity compared with free PSL and PSL immobilized on sole activated carbon or chitosan. The enantiomeric excess value of (S)-1-phenyl-3-buten-1-ol and (R)-1-phenyl-3-buten-1-ol acetate reached 99 and $94 \%$, respectively at $51 \%$ substrate conversion. Furthermore, the PSL/CS-AC catalyst possessed of higher thermal stability, re-usability and storage stability.

\section{EXPERIMENTAL}

Activated carbon, obtained from Aladdin (Shanghai, China). Chitosan (DACe $\geq 90 \%$ ) was obtained from Haidei Bei Marine Biological Engineering Co., Ltd. (Jinan, China). Pseudomonas cepacia Lipase (PSL) was supplied by Amano (USA). (R,S)-1-Phenyl-3-buten-1-ol was purchased from Sigma-Aldrich Shanghai Pvt. Ltd. (Shanghai, China). Heptane, isooctane, cyclohexane, toluene, benzene, acetonitrile, vinyl acetate and other analytical reagents were purchased from Kelong Chemicals (Chengdu, China). All chemicals and enzymes were used without any further purification.

Preparation and characterization of chitosan coatingmodified activated carbon: The activated carbon was modified by chitosan in order to improve its surface biocompatibility. Chitosan was dissolved in $1 \%(\mathrm{w} / \mathrm{w})$ aqueous acetic acid solution at a concentration of $0.1 \mathrm{~g} / 100 \mathrm{~mL}$ at $50{ }^{\circ} \mathrm{C}$. Dry activated carbon particles $(0.5,1.0$ and $1.5 \mathrm{~g})$ were added to chitosan solution and the resulting mixture was shaken for 1-2 h. After added 0.1 N sodium hydroxide, flocculent precipitate appeared from chitosan solution, the precipitate was obtained by filtration and washed with distilled water to remove residual sodium hydroxide. The chitosan-coated activated carbons CS-AC were dried overnight in a vacuum oven at $60{ }^{\circ} \mathrm{C}$.

The surface area and pore size distribution were derived from the $\mathrm{N}_{2}$ adsorption-desorption isotherms (Micromeritics ASAP-2010). Surface morphology of CS-AC was observed under a scanning electron microscope (SEM, JSM-7500F/ QUANTAX).

Pseudomonas cepacia lipase (PSL) immobilization on CS-AC: Pseudomonas cepacia lipase was immobilized onto the CS-AC as follows: $0.1 \mathrm{~g} \mathrm{CS}-\mathrm{AC}$ was dispersed into $10 \mathrm{~mL}$ isooctane followed by the addition of $30 \mathrm{mg}$ of PSL in solution. The mixture was shaken in a water bath for $4 \mathrm{~h}$ at $30^{\circ} \mathrm{C}$. The resulting products were separated, washed with $10 \mathrm{~mL}$ of isooctane and then dried under vacuum, denoted as PSL/CSAC.

Transesterification resolution of $(\mathrm{R}, \mathrm{S})-1$-phenyl-3buten-1-ol: $100 \mathrm{mg}$ of immobilized lipase PSL/CS-AC or $30 \mathrm{mg}$ of free lipase was firstly placed in a $25 \mathrm{~mL}$ apparatus, followed by the addition of $10 \mathrm{~mL}$ of heptane, $92 \mu \mathrm{L}$ of vinyl acetate and $0.25 \mathrm{mmol}$ of (R,S)-1-phenyl-3-buten-1-ol. Then the apparatus was oscillated in a $130 \mathrm{rpm}$ water bath shake bed at the reaction temperature. The principle of transesterification resolution of (R,S)-1-phenyl-3-buten-1-ol catalyzed by lipase is shown in the following equation:

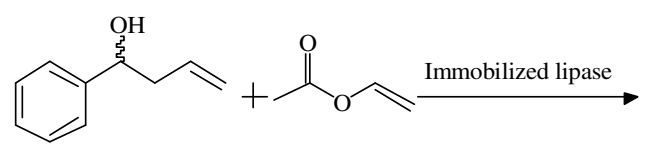

(R,S)-1-phenyl-3-buten-1-ol

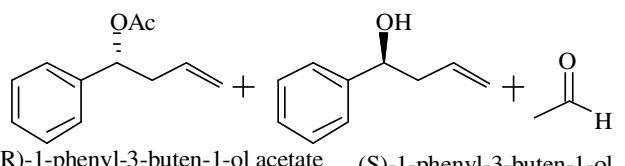

(R)-1-phenyl-3-buten-1-ol acetate (S)-1-phenyl-3-buten-1-ol

Reaction process and enantiomeric excess (ee) were monitored by periodic withdrawal of clear liquid samples from the reaction mixture which were analyzed by high performance gas chromatography (HPGC) (FL9790II, Fuli Analysis Instrument Co., Ltd., China) equipped with Cyclosil-B (Agilent technologies, China). The conversion $(\mathrm{C}, \%)$ was calculated from the enantiomeric excess of the substrate $\left(\mathrm{ee}_{\mathrm{s}}, \%\right)$ and product $\left(\mathrm{ee}_{\mathrm{p}}, \%\right)$ based on the following formulas:

$$
\begin{gathered}
\mathrm{C}(\%)=\frac{\mathrm{ee}_{\mathrm{S}}}{\mathrm{ee}_{\mathrm{S}}+\mathrm{ee}_{\mathrm{P}}} \\
\mathrm{ee}_{\mathrm{S}}(\%)=\frac{[\mathrm{S}]_{\mathrm{S}}-[\mathrm{R}]_{\mathrm{S}}}{[\mathrm{S}]_{\mathrm{S}}+[\mathrm{R}]_{\mathrm{S}}}, \mathrm{ee}_{\mathrm{P}}(\%)=\frac{[\mathrm{R}]_{\mathrm{P}}-[\mathrm{S}]_{\mathrm{P}}}{[\mathrm{R}]_{\mathrm{P}}+[\mathrm{S}]_{\mathrm{P}}}
\end{gathered}
$$

In the formulae, $[\mathrm{S}]_{\mathrm{S}}$ and $[\mathrm{R}]_{\mathrm{S}}$ denotes the concentration of (S)-1-phenyl-3-buten-1-ol and (R)-1-phenyl-3-buten-1-ol, respectively, also $[R]_{P}$ and $[S]_{P}$ denotes the concentration of (R)-1-phenyl-3-buten-1-ol ester and (S)-1-phenyl-3-buten-1ol ester, respectively.

\section{RESULTS AND DISCUSSION}

Characterizations of CS-AC: $\mathrm{N}_{2}$ adsorption-desorption isotherms of activated carbon and CS-AC with different mass ratios were showed in Fig. 1. With increasing chitosan amount, $\mathrm{m}$ (chitosan): $\mathrm{m}$ (activated carbon) from 1:15 to 1:5, the adsorption capacity of CS-AC composite gradually decreased (1:5, $1: 10,1: 15$ mean the mass ratio of chitosan and activated carbon). The surface morphologies of activated carbon with and without coating(s) were obtained by SEM (Fig. 2). The surface of uncoated activated carbon had a rough and highly porous structure. However, the surface of chitosan-coated activated carbon was greatly improved and much smoother than uncoated activated carbon.

Comparing the catalytic activity of immobilized lipase and free lipase: The catalytic activities of transesterification resolution of (R,S)-1-phenyl-3-buten-1-ol as a function of reaction time over the free and immobilized lipase were shown in Fig. 3. It indicated that immobilized lipase PSL/CS-AC exhibited much higher catalytic activity comparing with the free lipase. The conversion of (S)-1-phenyl-3-buten-1-ol reached $50 \%$ on PSL/CS-AC after $12 \mathrm{~h}$, however the conversion was only $6.5 \%$ on free lipase. It was worthy to mention that the enantioselectivity of (S)-1-phenyl-3-buten-1-ol was very high on PSL/CS-AC. After reaction $18 \mathrm{~h}$, the enantiomeric excesses (ee $)$ of (S)-1-phenyl-3-buten-1-ol were maintained at $99 \%$, thereby higher optical purity substrate (S)-1-phenyl3-buten-1-ol could be obtained. However, the enantiomeric

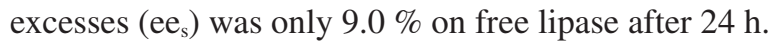

Free PSL was well dispersed during immobilization, which decreases the chance of free enzyme convergence in 


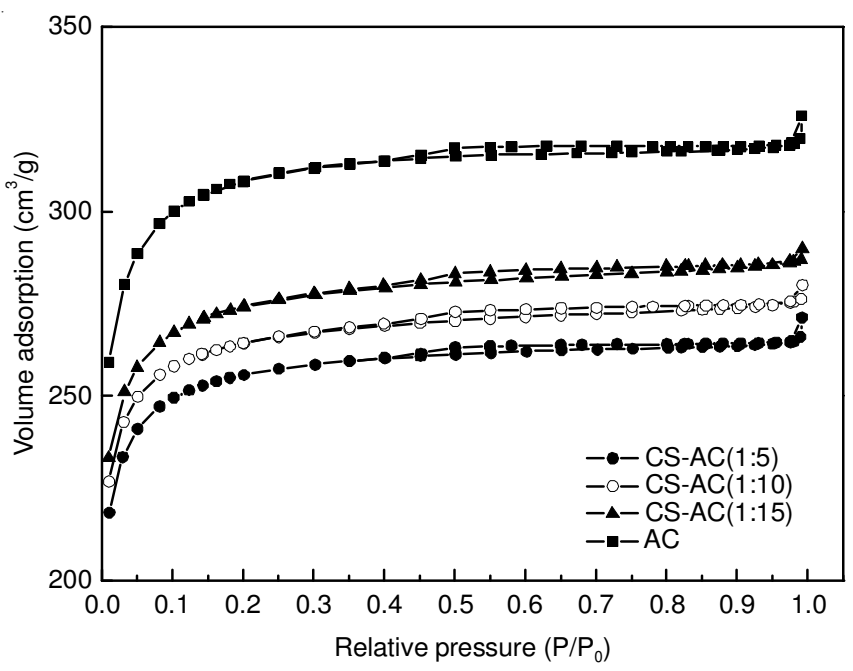

Fig. 1. $\mathrm{N}_{2}$ adsoption-desorption isotherms of activated carbon and CS-AC
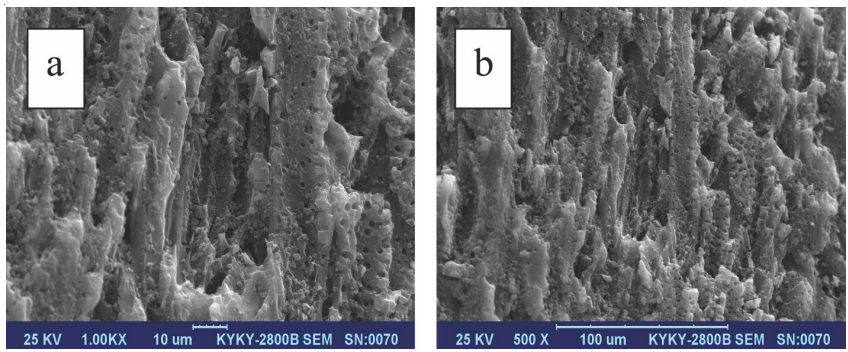

Fig. 2. SEM images of activated carbon (a) and CS-AC (1:10) (b)
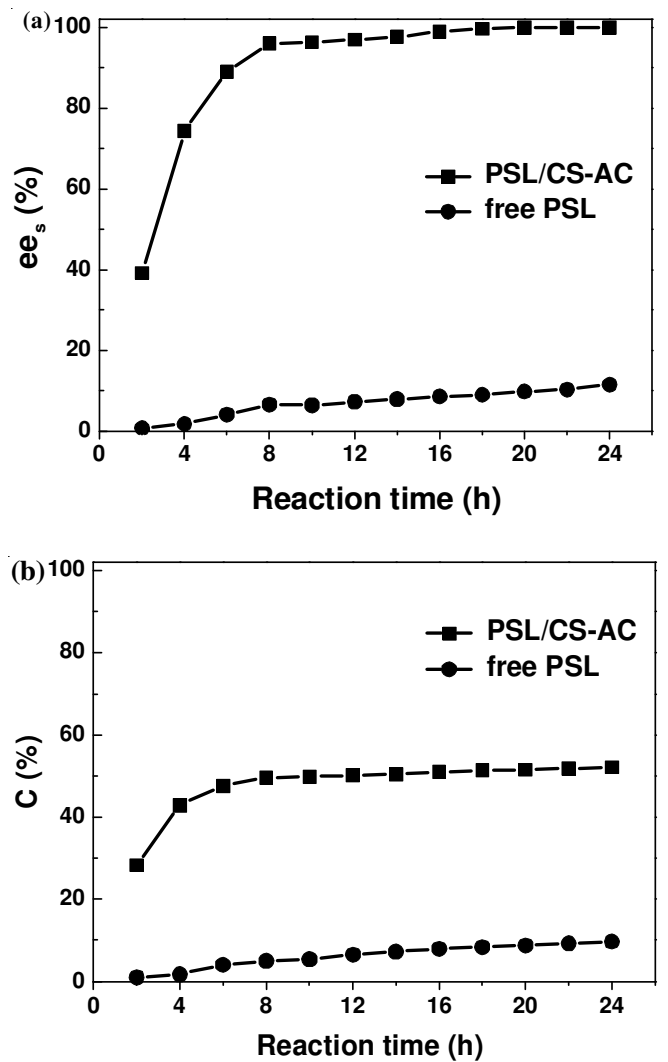

Fig. 3. Compared catalytic performance of PSL/CS-AC and free PSL

organic solvents. Comparing with the free PSL, the exposed active sites on immobilized PSL were much more, increasing the accessibility for substrate to the active site of enzyme and enhancing the activity of immobilized enzyme. A second possibility was that the conformation of lipase changed during the course of the CS-AC preparation, which possibly made the lipase adopt a more active conformation.

Comparing the catalytic activity of immobilized lipase on different supporters: Fig. 4 displayed the supporter effect on catalytic activity of immobilized lipase. The results showed that the enzyme activity of PSL/CS-AC was higher than that of PSL/CS and PSL/AC. For the immobilized lipase PSL/CSAC catalyzing transesterification resolution of racemic 1-phenyl3 -buten-1-ol, when reaction temperature was $40^{\circ} \mathrm{C}$ and reaction time was $6 \mathrm{~h}$, the ee values of (S)-1-phenyl-3-buten-1-ol and (R)-1-phenyl-3-buten-1-ol acetate reached, respectively 90.1 and $98.1 \%$ with the reaction substrate conversion of $47.9 \%$. It demonstrated that chitosan could improve the surface biocompatibility of activated carbon and provide a suitable surface for enzyme immobilization. Therefore, chitosan may have a widely potential application in preparation of the required functional carriers.

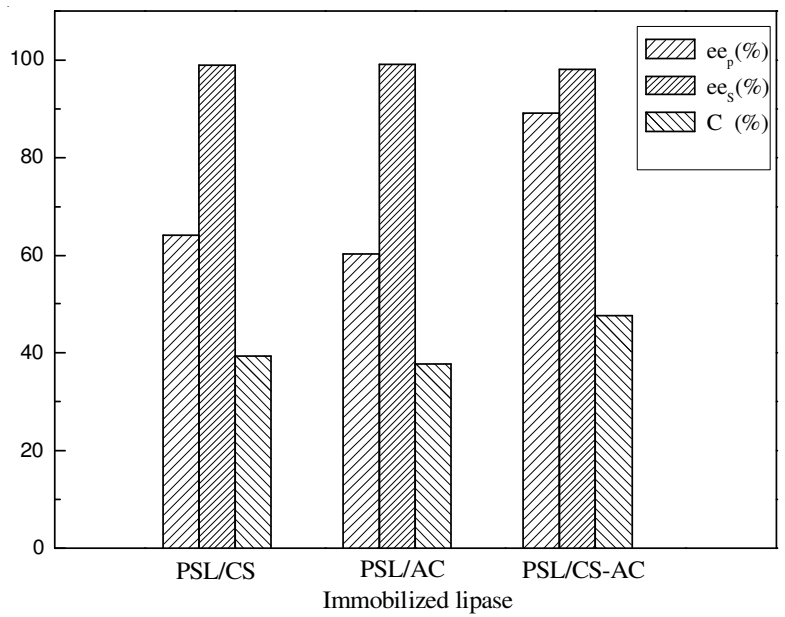

Fig. 4. Compared catalytic performance of immobilized lipase on different supporters

Recycle reusability of immobilized lipases PSL/CS-AC: The major and important purpose of immobilized lipases is in the design of reusable biocatalysts. Hence, we also investigated the durability of PSL/CS-AC in the repeated catalyzed transesterification of (R,S)-1-phenyl-3-buten-1-ol. After each run, the immobilized lipase was washed several times with heptane and was reintroduced into fresh reaction medium for another assay run. These procedures were repeated up to several times. Fig. 5 showed that the $e_{s}$ and $e_{p}$ values were only slightly decreased during 10 consecutive operations at $40{ }^{\circ} \mathrm{C}$ under the same reaction time of $18 \mathrm{~h}$. These results indicated that PSL/CS-AC could retain high enantioselectivity and catalytic performance in the process of reuse and the recycle reusability of PSL/CS-AC was good. However, it was still not ideal because the activity of immobilized lipase was decreased with increasing the recycle number, which may be due to the leakage and deactivation of partial lipase resulting from long-playing mechanical stirring under the continuous reactions.

Comparing the thermal stability of free PSL and immobilized lipases PSL/CS-AC: The transesterification of (R,S)-1-phenyl-3-buten-1-ol catalyzed by PSL/CS-AC in a 


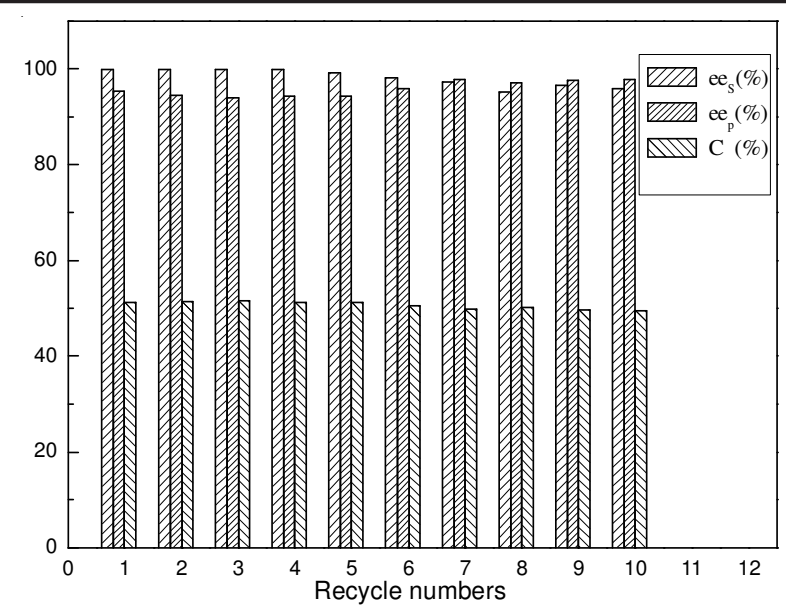

Fig. 5. Reusability of PSL/CS-AC

temperature range from 40 to $85^{\circ} \mathrm{C}$ was investigated in Table-1 and the highest activity of PSL/CS-AC was obtained at $40^{\circ} \mathrm{C}$. The thermal stability of the free and immobilized lipase was studied under the same temperature range. The enantiomeric excess of (S)-1-phenyl-3-buten-1-ol and (R)-1-phenyl-3-buten1-ol acetate reached, respectively 63.2 and $96.8 \%$ with the reaction substrate conversion of $40.0 \%$ when the reaction temperature was $85^{\circ} \mathrm{C}$. However, both conversion and enantiomeric excesses $\left(\mathrm{ee}_{\mathrm{s}}\right)$ were only $0.8 \%$ on the free lipase. It was concluded that the thermal stability of PSL could be greatly improved through the immobilization on CS-AC.

TABLE-1

THERMAL STABILITY OF FREE PSL AND PSL/CS-AC

\begin{tabular}{ccccc}
\hline $\begin{array}{c}\text { Reaction } \\
\text { temperature }\left({ }^{\circ} \mathrm{C}\right)\end{array}$ & Lipase & $\mathrm{ee}_{\mathrm{s}}^{\mathrm{a}}(\%)$ & $\mathrm{ee}_{\mathrm{p}}(\%)$ & $\mathrm{C}(\%)$ \\
\hline \multirow{2}{*}{40} & Free PSL & 1.7 & 99.9 & 1.7 \\
& PSL/CS-AC & 90.1 & 98.1 & 47.9 \\
\hline \multirow{2}{*}{70} & Free PSL & 1.1 & 99.9 & 1.1 \\
& PSL/CS-AC & 79.1 & 95.8 & 45.2 \\
\hline \multirow{2}{*}{80} & free PSL & 0.9 & 99.9 & 0.9 \\
& PSL/CS-AC & 70.0 & 97.7 & 41.6 \\
\hline \multirow{2}{*}{85} & Free PSL & 0.8 & 99.9 & 0.8 \\
& PSL/CS-AC & 63.2 & 96.8 & 40.0 \\
\hline
\end{tabular}

${ }^{a}$ Reaction conditions: reaction time $6 \mathrm{~h}$.

Storage stability of immobilized lipases PSL/CS-AC: Enzyme is generally stored at $0-4{ }^{\circ} \mathrm{C}$ to maintain the stability of its nature conformation. However, enzyme is inevitably exposed to external environment and loses activity rapidly in industrial application. Thus the storage stability of immobilized enzyme has always been of interest. As shown in Table-2, immobilized lipase PSL/CS-AC could remain high catalytic performance and enantioselectivity after storing for 90 days at room temperature, the enantiomeric excesses of (S)-1-phenyl3-buten-1-ol and (R)-1-phenyl-3-buten-1-ol acetate were still sustained at 94.1 and $99.7 \%$, respectively with the reaction substrate conversion of $47.4 \%$.

\begin{tabular}{cccc}
\hline \multicolumn{4}{c}{ TABLE-2 } \\
STORAGE STABILITY OF PSL/CS-AC \\
\hline Storage days $(\mathrm{d})$ & $\mathrm{ee}_{\mathrm{s}}{ }^{\mathrm{a}}(\%)$ & $\mathrm{ee}_{\mathrm{p}}(\%)$ & $\mathrm{C}(\%)$ \\
\hline 1 & 99.9 & 94.9 & 51.3 \\
90 & 94.1 & 99.7 & 47.4 \\
\hline
\end{tabular}

${ }^{a}$ Reaction conditions: reaction temperature $40^{\circ} \mathrm{C}$, reaction time $18 \mathrm{~h}$

\section{Conclusion}

In summary, we have successfully developed an efficient method for the transesterification resolution of racemic 1phenyl-3-buten-1-ol by PSL immobilized on chitosan-coated activated carbon. Chitosan was used as a modified material to improve the surface biocompatibility and increase the surface functional groups density of the activated carbon. The prepared immobilized lipase PSL/CS-AC catalyst exhibited much enhanced catalytic activity for resolving racemic 1-phenyl-3buten-1-ol to the corresponding optically pure (S)-1-phenyl3-buten-1-ol compared with the free lipase PSL and PSL immobilized on single activated carbon or chitosan. The ee and $e_{\mathrm{p}}$ reached 99 and $94 \%$, respectively with the reaction substrate conversion of $51 \%$. Moreover, the immobilized lipase PSL/CS-AC catalyst possessed of high thermal stability, reusability and storage stability.

\section{ACKNOWLEDGEMENTS}

This work was financially supported by the National Natural Science Foundation of China ( No.21263020) and the National Basic Research Program 973 (No. 2012CB723106).

\section{REFERENCES}

1. G.W. Zheng and J.H. Xu, Curr. Opin. Biotechnol., 22, 784 (2011).

2. R.N. Patel, Food Technol. Biotechnol., 42, 305 (2004).

3. A. Arefolov and J.S. Panek, Org. Lett., 4, 2397 (2002)

4. T.P. Loh, Q.Y. Hu and J.J. Vittal, Synlett., 523 (2000).

5. S. Singh, S. Kumar and S.S. Chimni, Tetrahedron Asymm., 13, 2679 (2002).

6. A. Ghanem and H.Y. Aboul-Enein, Tetrahedron, 15, 3331 (2004).

7. M. Nagarl, S.K. Dwivedi and D. Shrivastava, Int. J. Pharm. Res. Sci., 2, 631 (2013)

8. Q. Wu, P. Soni and M.T. Reetz, J. Am. Chem. Soc., 135, 1872 (2013).

9. R.C. Rodrigues, C. Ortiz, Á. Berenguer-Murcia, R. Torres and R. FernándezLafuente, Chem. Soc. Rev., 42, 6290 (2013).

10. S. Cantone, V. Ferrario, L. Corici, C. Ebert, D. Fattor, P. Spizzo and L. Gardossi, Chem. Soc. Rev., 42, 6262 (2013).

11. G. Yang, J. Wu, G. Xu and L. Yang, J. Mol. Catal. B, 57, 96 (2009).

12. A.S. Rani, M.L.M. Das and S. Satyanarayana, J. Mol. Catal. B, 10, 471 (2000).

13. A.G. Kumar, S. Swarnalatha, P. Kamatchi and G. Sekaran, Biochem. Eng. J., 43, 185 (2009).

14. J.C. Naranjo, A. Córdoba, L. Giraldo, V.S. García and J.C. MorenoPiraján, J. Mol. Catal. B, 66, 166 (2010).

15. J. Miao, F. Zhang, M. Takieddin, S. Mousa and R.J. Linhardt, Langmuir, 28, 4396 (2012).

16. K. Ramani, S. Karthikeyan, R. Boopathy, L.J. Kennedy, A.B. Mandal and G. Sekaran, Process Biochem., 47, 435 (2012).

17. B. Krajewska, Enzyme Microb. Technol., 35, 126 (2004).

18. V.R. Sinha, A.K. Singla, S. Wadhawan, R. Kaushik, R. Kumria, K. Bansal and S. Dhawan, Int. J. Pharm., 274, 1 (2004). 